\title{
Alteration in P-glycoprotein Functionality Affects Intrabrain Distribution of Quinidine More Than Brain Entry-A Study in Rats Subjected to Status Epilepticus by Kainate
}

\author{
Stina Syvänen, ${ }^{1,3}$ Maarten Schenke, ${ }^{1,2}$ Dirk-Jan van den Berg, ${ }^{1}$ Rob A. Voskuyl, ${ }^{1,2}$ and Elizabeth C. de Lange ${ }^{1}$
}

Received 15 September 2011; accepted 15 December 2011; published online 4 January 2012

\begin{abstract}
This study aimed to investigate the use of quinidine microdialysis to study potential changes in brain P-glycoprotein functionality after induction of status epilepticus (SE) by kainate. Rats were infused with 10 or $20 \mathrm{mg} / \mathrm{kg}$ quinidine over $30 \mathrm{~min}$ or $4 \mathrm{~h}$. Plasma, brain extracellular fluid (brain ECF), and endof-experiment total brain concentrations of quinidine were determined during $7 \mathrm{~h}$ after the start of the infusion. Effect of pretreatment with tariquidar $(15 \mathrm{mg} / \mathrm{kg}$, administered $30 \mathrm{~min}$ before the start of the quinidine infusion) on the brain distribution of quinidine was assessed. This approach was repeated in kainate-treated rats. Quinidine kinetics were analyzed with population modeling (NONMEM). The quinidine microdialysis assay clearly revealed differences in brain distribution upon changes in Pglycoprotein functionality by pre-administration of tariquidar, which resulted in a 7.2-fold increase in brain ECF and a 40-fold increase in total brain quinidine concentration. After kainate treatment alone, however, no difference in quinidine transport across the blood-brain barrier was found, but kainatetreated rats tended to have a lower total brain concentration but a higher brain ECF concentration of quinidine than saline-treated rats. This study did not provide evidence for the hypothesis that Pglycoprotein function at the blood-brain barrier is altered at 1 week after SE induction, but rather suggests that P-glycoprotein function might be altered at the brain parenchymal level.
\end{abstract}

KEY WORDS: blood-brain barrier; epilepsy; microdialysis; P-glycoprotein (P-gp); quinidine.

\section{INTRODUCTION}

P-glycoprotein (P-gp) is the best studied efflux transporter at the endothelial capillaries constituting the bloodbrain barrier $(\mathrm{BBB})(1,2)$. It is responsible for reduced brain distribution of many clinically used drugs. For drugs aimed at systemic targets this can be a benefit as the action of P-gp will help to reduce central side effects. P-gp might, however, also hamper the ability of drugs to reach target sites within the brain. For example, about $30-40 \%$ of all people with epilepsy do not become fully seizure free with current medications, even when treated at maximal tolerated doses. Lack of response in these individuals is not limited to a specific drug or drug class, but occurs with the complete range of antiepileptic drugs $(3,4)$. Thus, it has been hypothesized that

\footnotetext{
${ }^{1}$ Division of Pharmacology, LACDR, Leiden University, P.O. Box 9502, 2300 RA Leiden, The Netherlands.

${ }^{2}$ Epilepsy Institute of The Netherlands Foundation (SEIN), P.O. Box 21, 2100 AA Heemstede, The Netherlands.

${ }^{3}$ To whom correspondence should be addressed. (e-mail: s.syvanen@ lacdr.leidenuniv.nl)

ABBREVIATIONS: BBB, Blood-brain barrier; ECF, Extra cellular fluid; ip, Intraperitoneal; OFV, Objective function value; P-gp, P-glycoprotein; PET, Positron emission tomography; SE, Status epilepticus; TQD, Tariquidar.
}

increased P-gp expression in a subpopulation of people with severe epilepsy is, at least in part, responsible for pharmacoresistance by impairing drug access to the brain

There are many methods used to study drug distribution into the brain (5). However, most of these methods focus on total brain concentrations. Since brain tissue is rather lipophilic, total brain concentrations will often mainly reflect the lipophilicity of the drug, i.e., a lipophilic drug will have a higher total brain concentration than a more hydrophilic drug. If the transport across the BBB is of interest, methods that measure free concentrations in brain are needed, as a reflection of the fraction of the molecules in plasma that can pass the tightly connected cell membrane of the BBB. Microdialysis is the state-of-the-art methodology for measuring free drug concentrations in the brain. Transport processes at the BBB and the brain parenchyma can be separated by using microdialysis in combination with measurement of plasma and total brain concentrations $(6,7)$.

Quinidine, a substrate with high affinity for P-gp, was used in the present study as a model substrate for determining P-gp functionality at the BBB. To investigate the specific contribution of P-gp at the BBB, rats were studied without or with co-administration of the $\mathrm{P}$-gp inhibitor tariquidar (TQD). Quinidine concentrations were determined in arterial plasma and extracellular brain fluid for $7 \mathrm{~h}$ after the start of quinidine administration. In addition, quinidine concentration in brain tissue was determined at the end of the experiment. 
In rats, treatment with intraperitoneal (ip) injections of kainate leads to status epilepticus (SE) (8). After a latent period of 3-4 weeks post-SE, rats start to show spontaneous seizures. The kainate model in rats displays several features of temporal lobe epilepsy, the most common form of epilepsy frequently associated with pharmacoresistance, including an increase in P-gp expression $(9,10)$. In a recent positron emission tomography (PET) study it was shown that the brain distribution volume of the PET P-gp substrate tracer $(R)-\left[{ }^{11} \mathrm{C}\right]$ verapamil was increased in kainate-treated rats compared to control rats (11). However, unlike microdialysis, PET measures total concentrations and hence pharmacokinetics at the level of the BBB versus intrabrain cannot be separated.

The aim of the present study was first develop a microdialysis method for studies of drug transport across the $\mathrm{BBB}$ and intrabrain distribution, and second, to investigate the influence of kainate treatment and TQD pre-administration on quinidine pharmacokinetics with focus on P-gp functionality, both at the BBB and brain parenchyma.

\section{MATERIALS AND METHODS}

\section{Chemicals and Solutions}

Tariquidar (XR9576, TQD) was obtained from Xenova Group PLC (Cambridge, England) or API Services Inc. (Westford, USA), kainic acid, quinidine, quinidine sulfate dehydrate, quinidine hemi sulfate and quinine hemi sulfate from Sigma Aldrich (Zwijndrecht, The Netherlands), triethyl amine from Baker (Deventer, The Netherlands), boric acid and orthophosphoric acid $85 \%$ was from Merck (Darmstadt, Germany), methyl tert-butyl etherfrom Bioslove (Valkenswaard, The Netherlands), isoflurane from Pharmachemie BV (Haarlem, The Netherlands), 5\% glucose in saline and saline from hospital pharmacy of the University Medical Centre Leiden (UMCL, Leiden, The Netherlands).

Perfusion fluid for microdialysis was a modified artificial cerebrospinal fluid solution previously described by Moghaddam et al. (12). In short, it was a water based solution containing $140 \mathrm{mM} \mathrm{NaCl}, 3 \mathrm{mM} \mathrm{KCl}, 1 \mathrm{mM}$ $\mathrm{MgCl}_{2}$, and $1.2 \mathrm{mM} \mathrm{CaCl}$. For injections, kainate was dissolved in saline to a total concentration of $5 \mathrm{mg} / \mathrm{mL}$; quinidine was dissolved in saline to a total concentration of 1.25 or $2.5 \mu \mathrm{g} / \mu \mathrm{L}$ and TQD was dissolved in $5 \%$ glucose in saline to a total concentration of $3.75 \mathrm{mg} / \mathrm{mL}$.

For the HPLC analysis, quinidine was dissolved in methanol and diluted with water to a concentration of
$100 \mu \mathrm{g} / \mathrm{mL}$. This stock solution was further diluted in water (plasma and brain analysis) or perfusion fluid (microdialysate analysis) to construct calibration curves for quinidine analysis.

\section{Animals}

Adult male Sprague-Dawley rats ( $n=74$, Harlan, Horst, The Netherlands) weighing 200-249 g on arrival were housed in groups of five to six per cage until treatment or surgery and thereafter housed individually. They were kept at a constant temperature of $21 \pm 1^{\circ} \mathrm{C}$ and at a $12 \mathrm{~h}$ light/dark cycle, in which white lights were switched on at 8:00 AM. Animals had unrestricted access to food (RM3 (E) DU, Special Diets Services B.V., Witham, Essex, England) and acidified water. Animal procedures were performed in accordance with Dutch laws on animal experimentation. All experiments were approved by the Ethics Committee for Animal Experiments of Leiden University (approval numbers UDEC08134 and UDEC08200).

Two groups of animals were studied: (1) untreated control rats and (2) kainate-treated rats. The number of animals in each group is shown in Table I. Of the initial 74 animals, 10 animals had to be excluded either because of technical reasons such as clogging of cannulae or irregularities in flow through the microdialysis probe, or due to death or insufficient recovery after kainate treatments.

The control group $(n=48)$ underwent surgery approximately after 1 week of habituation and 7 days prior to microdialysis experiments. In short, rats were anesthetized via a nose mask with isoflurane, $4-5 \%$ induction and $1.5-2 \%$ maintenance, in oxygen $1 \mathrm{~L} / \mathrm{min}$. Blood cannulae (Portex Fine bore PE tubing, Smith Medical B.V., Rosmalen, The Netherlands) were implanted in the femoral artery for blood sampling and the femoral vein for drug administration. This was followed by insertion of a microdialysis guide with a dummy probe (CMA, Solna, Sweden), which was implanted in the hippocampus (AP-5.6, L4.6, V-2.5). Six of the control animals were used to determine the in vivo recovery of quinidine by retro dialysis (13). These animals had a microdialysis guide and dummy implanted 7 days prior to the experiment, but no blood cannulae.

The rats in group 2, were treated with saline $(n=3)$, or kainic acid $(n=16)$ to induce SE after approximately 1 week of habituation and 7 days prior to microdialysis experiments. Kainic acid was administered repetitively until full-blown seizures were seen (8). First, an initial dose of $10 \mathrm{mg} / \mathrm{kg}$ $(2 \mathrm{~mL} / \mathrm{kg})$ i.p. was administered followed by $5 \mathrm{mg} / \mathrm{kg}(1 \mathrm{~mL} / \mathrm{kg})$ i.p. every $30-60 \mathrm{~min}$ until stage IV seizures according to Racine's

Table I. Number of Animals in the Different Treatment Groups

\begin{tabular}{|c|c|c|c|c|}
\hline & $\begin{array}{l}\text { Control rats }+ \text { vehicle } \\
\text { co-administration }\end{array}$ & $\begin{array}{l}\text { Control rats }+ \text { tariquidar } \\
\text { co-administration }\end{array}$ & $\begin{array}{l}\text { Kainate-treated + vehicle } \\
\text { co-administration }\end{array}$ & $\begin{array}{l}\text { Kainate-treated + tariquidar } \\
\text { co-administration }\end{array}$ \\
\hline Quinidine $12.5 \mu \mathrm{g} / \mathrm{min}$ over $4 \mathrm{~h}$ & 7 & 6 & - & - \\
\hline Quinidine $25.0 \mu \mathrm{g} / \mathrm{min}$ over $4 \mathrm{~h}$ & 8 & 8 & - & - \\
\hline Quinidine $100 \mu \mathrm{g} / \mathrm{min}$ over $30 \mathrm{~min}$ & $9^{\mathrm{a}}$ & $7^{\mathrm{b}}$ & 8 & 8 \\
\hline In vivo retrodialysis & 3 & 3 & - & - \\
\hline
\end{tabular}

${ }^{a}$ Two of the nine animals were included from the second groups of rats (see text for further explanation)

${ }^{b}$ One of the seven animals were included from the second groups of rats (see text for further explanation) 
scale (14) occurred, or when a total amount of $30 \mathrm{mg} / \mathrm{kg}$ kainic acid was reached. Six days later and 1 day prior to the microdialysis experiment, the animals underwent cannulation surgery and the implantation of the microdialysis probe as described in the section above.

After the microdialysis study, animals were euthanized by decapitation and the brain was removed. All samples, i.e., plasma, microdialysate and brain tissue were stored at $-80^{\circ} \mathrm{C}$ until analysis

\section{Pharmacokinetic Study in Rats}

The dummy probe was replaced with the microdialysis probe 1 day (18-22 h) prior to the microdialysis experiment. On the day of the microdialysis study, the probe inlet was connected to the perfusion fluid syringe and the outlet to an automatic cooled fraction collector (Univentor microsampler 820, Univentor Ltd, Zejtun, Malta). When the rat was properly connected the perfusion rate was set to $2 \mu \mathrm{L} / \mathrm{min}$ and was kept on this rate throughout the experiment. Samples were collected for 15 min, i.e., the fraction collector turned every $15 \mathrm{~min}$. The perfusion rate was checked by weighing each sample, and samples obtained at perfusion rates deviating from the set rate with more than $10 \%$ were excluded. The probes were perfused for at least $60 \mathrm{~min}$ (blank period) prior to the start of the quinidine intravenous infusion. Quinidine was infused at $10 \mu \mathrm{L} / \mathrm{min}(2.5$ or $1.25 \mu \mathrm{g} / \mu \mathrm{L})$ over $4 \mathrm{~h}$ or $40 \mu \mathrm{L} / \mathrm{min}(2.5 \mu \mathrm{g} / \mu \mathrm{L})$ over $30 \mathrm{~min}$. The number of animals in each dose group is shown in Table I. Half of the animals were treated with the P-gp inhibitor TQD, $15 \mathrm{mg} / \mathrm{kg}, 30 \mathrm{~min}$ before the start of the quinidine infusion. The other half was treated with $5 \%$ glucose in saline (vehicle) at the same time point.

\section{In Vivo Retro Dialysis}

Microdialysis probe recovery was determined in six rats through in vivo retro dialysis (15). The animals were each infused with one or two concentrations of quinidine in perfusion fluid. The quinidine concentrations were randomized and ranged between 20 and $500 \mathrm{ng} / \mathrm{mL}$ to cover the whole concentration range observed in the study. The probe recovery was calculated as described in Eq. 1.

$$
\text { Extraction fraction }=\frac{C_{\mathrm{in}}-C_{\mathrm{out}}}{C_{\mathrm{in}}}
$$

Where $C_{\text {in }}$ is the quinidine concentration in the perfusion fluid, $C_{\text {out }}$ is the concentration in the dialysate and the "extraction fraction" loss of molecules to the tissue. It is assumed that the loss is equal to the gain, i.e., if $25 \%$ of molecules in the perfusion fluid are lost to the tissue it is assumed that a blank perfusion fluid, as used in the experiments, will pick up $25 \%$ of the molecules present in the tissue.

\section{Quantification of Quinidine in Plasma, Dialysates, and Brain Tissue}

Quinidine was measured using HPLC with fluorescence detection. A LC10-ADVP HPLC pump (Shimadzu, 's-Hertogenbosch, the Netherlands) using a flow of $1 \mathrm{~mL} / \mathrm{min}$. An Altima C-18 150 $\times 4.6 \mathrm{~mm}$ column (Grace Altech, Breda, the
Netherlands) was used to elute quinidine. Detection was performed using a Jasco FP-1920 fluorescence detector (Jasco, de Meern, the Netherlands) set at $488 \mathrm{~nm}$ for excitation and $512 \mathrm{~nm}$ for emission. Injection was performed using a Waters 717 autosampler (Waters, Etten-Leur, the Netherlands). The injection volume was $20 \mu \mathrm{L}$. Data acquisition was performed using Empower Software (Waters, Etten-Leur, The Netherlands). The mobile phase consisted of acetonitrile and phosphate buffer $(25 \mathrm{mM})$ with triethyl amine $(10 \mathrm{mM})$ at a $\mathrm{pH}$ of 2.3 in a ratio of 17:83 $(v / v)$ and 14/86 $(v / v)$ for microdialysis and plasma samples, respectively.

Microdialysate was injected directly, without any pretreatment and measured dialysate concentrations were converted into brain extracellular fluid (brain ECF) concentrations by division with the extraction fraction obtained using Eq. 1.

$$
C_{\mathrm{ECF}}=\frac{C_{\text {dial }}}{\text { Extraction fraction }}
$$

To $20 \mu \mathrm{L}$ plasma, $50 \mu \mathrm{L}$ of the internal standard quinine (Sigma Aldrich, Zwijndrecht, the Netherlands) was added in a concentration of $500 \mathrm{ng} / \mathrm{mL}$.

After homogenization with $200 \mu \mathrm{L}$ of borate buffer $\mathrm{pH} 10,5 \mathrm{~mL}$ of methyl tert-butyl ether was added. After vortexing, centrifugation, and freezing of the aqueous layer, the organic phase was evaporated to dryness. The extracts were reconstituted in $100 \mu \mathrm{L}$ of mobile phase and centrifuged at $4,000 \times g$ during $5 \mathrm{~min}$. The clean plasma extracts were injected using a mobile phase with an acetonitrile/buffer ratio of 1:6. Calibration was performed using $20 \mu \mathrm{L}$ aliquots of quinidine in concentrations of $5,10,20,50,100,200,500$, $1,000,2,000$, and $5,000 \mathrm{ng} / \mathrm{mL}$ which were added to $20 \mu \mathrm{L}$ blank plasma.

Quinidine concentration in brain tissue was analyzed by the following steps: whole brain was homogenized in phosphate buffer at $\mathrm{pH}$ 7.4. Per gram brain, $5 \mathrm{~mL}$ of buffer was added $(50 \mathrm{mM})$. After addition of the internal standard quinine to $0.6 \mathrm{~mL}$ of the homogenate, $100 \mu \mathrm{L}$ of sodium hydroxide $1 \mathrm{M}$ was added and mixed thoroughly. Tertiary butyl methyl ether was added in a volume of $5 \mathrm{~mL}$. After vortexing for $5 \mathrm{~min}$ and centrifugation, $4 \mathrm{~mL}$ of the supernatant was transferred to a clean glass tube and $100 \mu \mathrm{L}$ of phosphoric acid $30 \mathrm{mM}$ was added. After another $5 \mathrm{~min}$ of vortexing followed by centrifugation at $4,000 \times \mathrm{g}$ for $10 \mathrm{~min}$, the supernatants were aspirated and discarded. The remaining aqueous phase was centrifuged for $10 \mathrm{~min}$ at $11,000 \times g$. An aliquot of $40-60 \mu \mathrm{L}$ was transferred to clean glass vials and $20 \mu \mathrm{L}$ was introduced into the HPLC system, using a mobile phase with an acetonitrile/buffer ratio of 1:6.

Precision and accuracy of the determination of quinidine in plasma and brain were below the $15 \%$ level. The precision and accuracy of quinidine measurement in microdialysate were less than $10 \%$. Limit of quantification was $5 \mathrm{ng} / \mathrm{mL}$ for plasma, $20 \mathrm{ng} / \mathrm{mL}$ for brain, and $1 \mathrm{ng} / \mathrm{mL}$ for dialysate.

\section{Data Analysis}

Analysis of quinidine pharmacokinetics was performed using nonlinear mixed effects modeling in NONMEM VI (GloboMax LLC, Hanover, MD, USA). The phamacokinetic model was constructed in three steps. In the first step, a pharmacokinetic model for quinidine plasma concentrations 
was developed. One, two, and three compartment models were evaluated. In the second step the model was extended to include brain ECF concentrations, while allowing the plasma estimates to change freely from the values obtained in the first step. Again, one and two compartment models were evaluated. The final step was to also include the total brain concentrations that were obtained at the end of the experiments.

Data from all rats were processed simultaneously. The subroutine ADVAN 6 and first-order conditional estimation with interaction were used throughout the modeling procedure. Model selection was based on the objective function value $(\mathrm{OFV})$ with the lowest value corresponding to the best model. For nested models, OFV reductions of 3.83, 6.63 and 10.83 units correspond to improved fits at $p<0.05, p<0.01$, and $p<0.001$ levels, respectively. Further, model parameter uncertainty and visual analysis using software Xpose 4 (16) implemented in $\mathrm{R}$ 2.7.1 (The $\mathrm{R}$ foundation for Statistical Computing) accessed from Census (17) was used to assess the model performance. For example, predicted concentrations were plotted against measured concentrations and conditional weighted residuals (18) were calculated and plotted against time and concentration.

The inter-individual variation of a parameter was described by the exponential variance model:

$$
\theta_{i}=\theta_{\mathrm{pop}} \cdot \exp \left(\eta_{i}\right)
$$

where $\theta_{i}$ is the parameter in the $i$ th animal, $\theta_{\text {pop }}$ the parameter in a typical animal and $\eta_{i}$ the inter-animal variability, which is assumed to be normally distributed around zero with a standard deviation $\omega$. Equation 3 provides a means to distinguish the parameter value for the $i$ th animal from the typical value predicted from the regression model. Inter-individual variation was investigated for all parameters, but incorporated only for those parameters for which it significantly ( $p<0.01$, OFV reduction of 6.63 units) improved the model.

Pre-administration (TQD or vehicle), rat group (control or kainate-treated), infusion time, quinidine dose, recovery time since surgery and animal weight on the experimental day were defined as covariates to study their effects on the parameter estimates. A stepwise forward addition and backward deletion approach was applied to test the significance $(p<0.01)$ for covariate inclusion. The residual variability was described with proportional error models for each compartment.

Performance of the population pharmacokinetic model was evaluated using 100 additional bootstrap replicates (re-sampling with replacement) of the data by fitting the final model to them.

\section{RESULTS}

\section{Quinidine Plasma and Brain Profiles}

Quinidine plasma and brain ECF concentration profiles for the groups of rats that were infused with $100 \mu \mathrm{g} / \mathrm{min}$ over $30 \mathrm{~min}$ are shown in Fig. 1. The brain uptake of quinidine without TQD pretreatment was in general low but was increased by pretreatment with TQD.
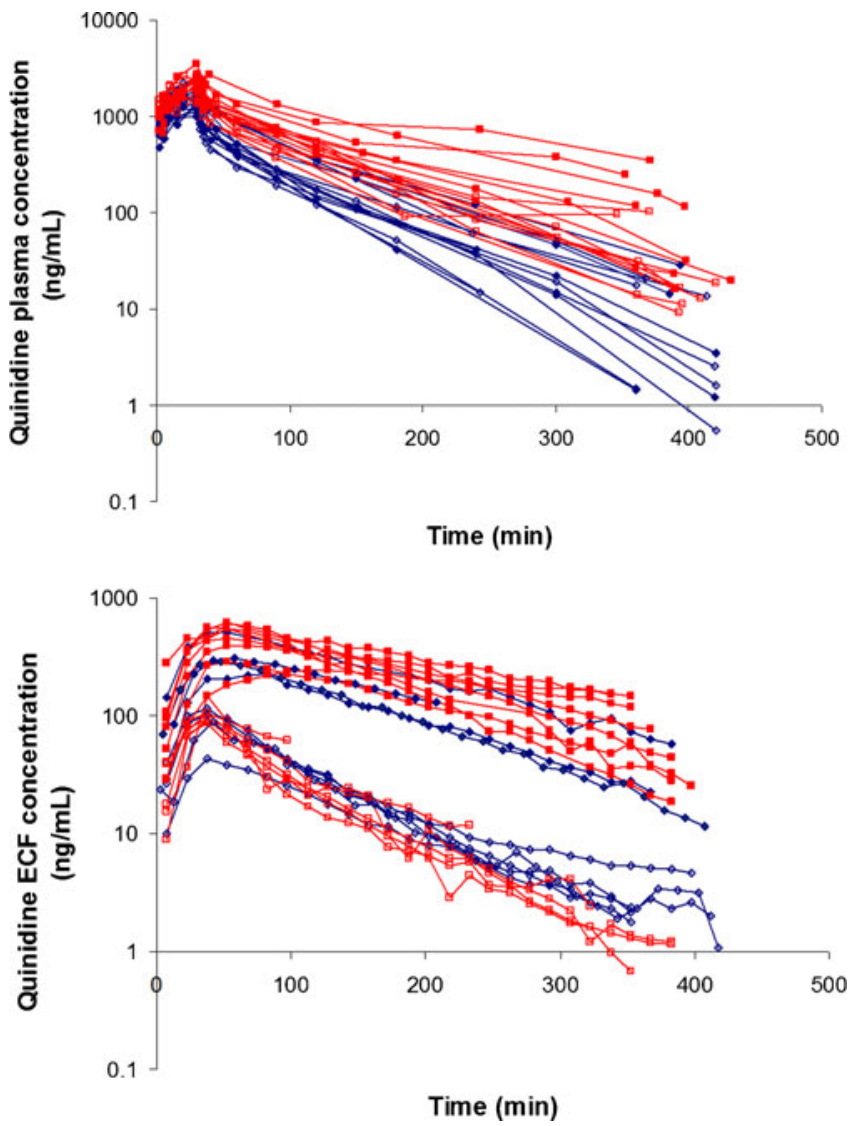

Fig. 1. Quinidine concentrations in plasma (upper panel) and brain ECF (lower panel) during and after an intravenous infusion of $100 \mu \mathrm{g} / \mathrm{min}$ over $30 \mathrm{~min}$. Blue and red symbols are saline- and kainate-treated rats, respective. Open and closed symbols are without and with tariquidar treatment, respectively

\section{Development of the Pharmacokinetic Model}

Quinidine kinetics in both plasma and brain were analyzed using mixed effects modeling in NONMEM. The final model including covariate effects is shown in Fig. 2. Both the plasma and brain profiles were best described with two compartment models. As a first step, only plasma was modeled and as a second step the brain ECF and total brain compartments were included. When the ECF and total brain compartments were included, only minor changes were observed for the plasma parameter estimates (Table II). The model diagnostics plots, separated for plasma and brain ECF concentrations, are shown in Fig. 3.

It was not possible to estimate $Q_{\text {in }}, Q_{\text {out }}$, and $V_{\text {br }}$ (Fig. 2) independently with good precision and thus the model was simplified to include a correlation between $Q_{\text {in }}$ and $Q_{\text {out }}$ :

$$
Q_{\text {in }}=f_{1} \cdot Q_{\text {out }}
$$

where $f_{1}$ describes the correlation between $Q_{\text {out }}$ and $Q_{\text {in }}$. In the final model, $Q_{\text {out }}$ was fixed to a value of $10.8 \mathrm{~mL} / \mathrm{min}$ as it was observed in the bootstrap analysis that the volume of distribution $V_{\mathrm{br} 1}$ and $Q_{\text {out }}$ were to some extent correlated, i.e. the ratio between the two parameters was stable, but for some of the data sets generated in the bootstrap analysis $V_{\mathrm{br} 1}$ was estimated to an unrealistically high value. The value of 


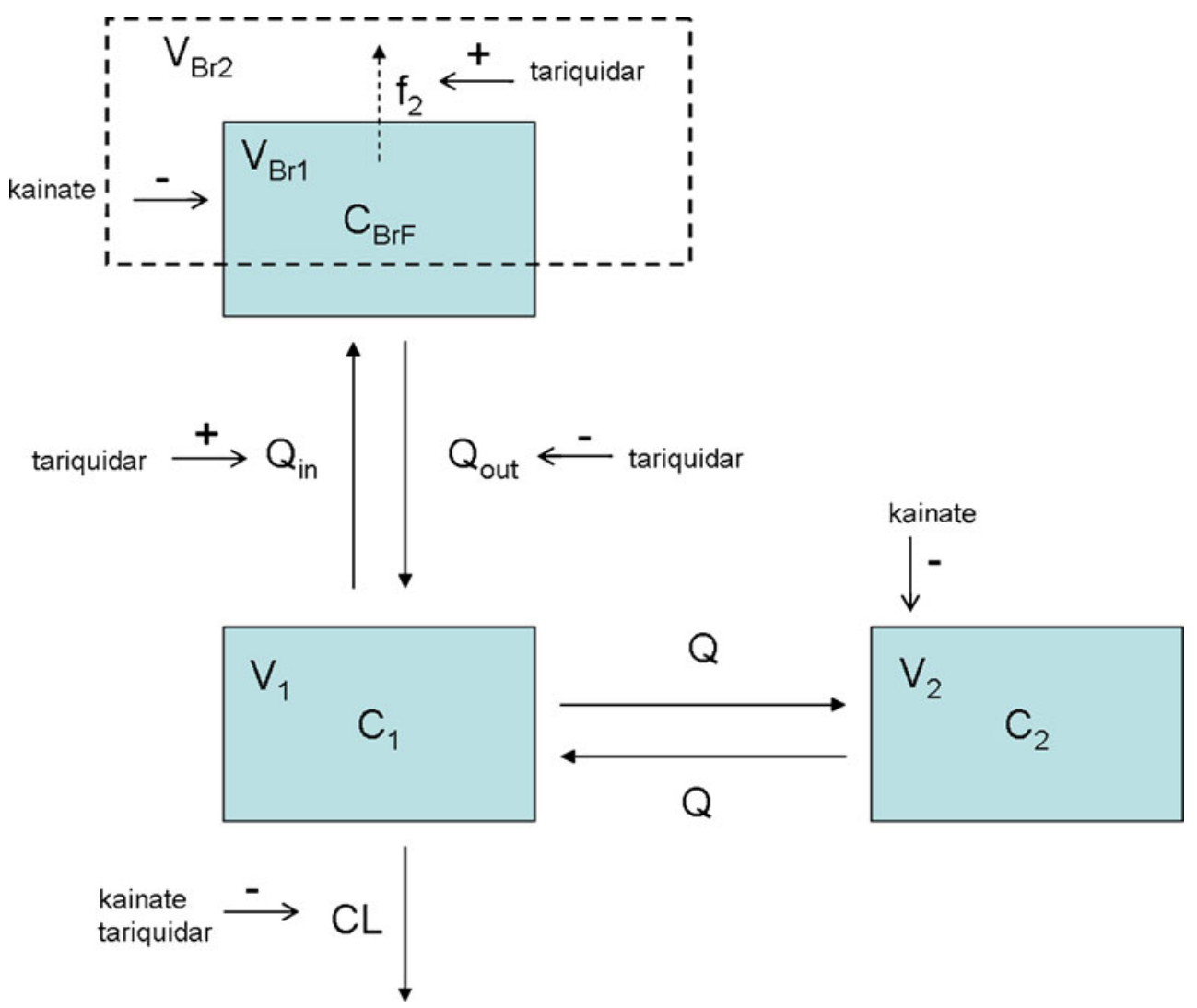

Fig. 2. Final pharmacokinetic model including covariates. $C_{1}, C_{2}, C_{\mathrm{BrF}}$, and $C_{\mathrm{BrT}}$ are the quinidine concentrations (in nanograms per milliliter) in central plasma, peripheral tissue, unbound brain, and deeper brain compartment, respectively. $V_{1}, V_{2}$, and $V_{\mathrm{Br}}$ are the volumes of distribution (in milliliters) in central plasma, peripheral tissue and unbound brain, respectively. CL, $Q, Q_{\text {in }}$, and $Q_{\text {out }}$ are the clearances (in milliliters per minute) from plasma, between the two plasma compartments, into the brain and out from the brain, respectively. $Q_{\text {in }}$ was defined as a fraction of $Q_{\text {out }}, Q_{\text {in }}=f_{1} \bullet Q_{\text {out }}$. The distribution from the free brain compartment to the deeper brain compartment was described with a fraction, $f_{2}$, so that $C_{\mathrm{BrT}}=f_{2} \bullet C_{\mathrm{BrF}}$. The covariate analysis showed that kainate treatment decreased the systemic clearance, the volume of distribution in peripheral tissue and brain while tariquidar co-administration decreased the systemic clearance, increased the quinidine clearance into the brain, decreased the clearance out from the brain and increased the distribution from the unbound brain compartment to the deeper brain compartment

$10.8 \mathrm{~mL} / \mathrm{min}$ was obtained with the final model when $Q_{\text {out }}$ was kept free during the estimation.

The concentration in the total brain compartment $\left(C_{\mathrm{br} 2}\right)$ was assumed to be correlated with the concentration in the first brain compartment $\left(C_{\mathrm{br} 1}\right)$ as:

$$
C_{\mathrm{br} 2}=f_{2} \cdot C_{\mathrm{br} 1}
$$

In the present study, measurements were obtained in both of these brain compartments. The concentration in the first brain compartment $\left(C_{\mathrm{br} 1}\right)$ was the free quinidine concentration in brain ECF, while the concentration in the second brain compartment $\left(C_{\mathrm{br} 2}\right)$ was the concentration of quinidine in total brain tissue, i.e., intra- and extracellular fluid as well as brain tissue.

Inter-individual variation was found for structural model parameters $\mathrm{CL}, V_{1}, V_{2}, V_{\mathrm{Br}}$, and $Q_{\text {in }}$ (Table II). The covariate analysis showed no correlation between parameter estimates and quinidine dose, infusion time, recovery time after surgery or recovery time after probe insertion. When only plasma was fitted the OFV was reduced by 9.5 units $(p<0.01)$ when animal weight was added as a covariate for clearance. However this covariate was not significant when the model was extended also to include brain compartments (Table III).

\section{Analysis of Influence of TQD and Kainate Treatment}

Both TQD pre-administration and kainate treatment were treated as categorical covariates, i.e., they were assigned a value of 0 or 1 . The final parameter equations, including covariates were defined as:

$$
\theta_{i}=\theta_{\text {pop }} \cdot \exp \left(\eta_{j}\right) \cdot \theta_{\text {covar }} \text { COVARIATE }(1 \text { or } 0)
$$

Where $\theta_{\text {covar }}$ describes the influence of TQD preadministration or kainate treatment on parameter estimate $\theta_{\text {pop }}$ (Eq. 6). In Eq. 6, the exponent COVARIATE was assigned a value of 1 for TQD pre-administration and kainate treatment and a value of 0 for vehicle co-administration and control rat. The $\theta_{\text {covar }}$ estimate therefore represents the fractional change between TQD and vehicle pre-administered or kainate-treated and untreated groups. Equations for all model parameters are given in Table IV. 
Table II. Quinidine Pharmacokinetic Model Parameter Estimates

\begin{tabular}{|c|c|c|c|c|c|c|c|}
\hline \multirow{3}{*}{$\begin{array}{l}\text { Objective function } \\
\text { Parameter }\end{array}$} & \multicolumn{3}{|c|}{ Estimation } & \multicolumn{2}{|c|}{ Bootstrap } & \multicolumn{2}{|c|}{ Estimation plasma only } \\
\hline & \multicolumn{3}{|c|}{-2898} & \multicolumn{2}{|c|}{-2964} & \multicolumn{2}{|c|}{-695} \\
\hline & Value & $\mathrm{CV} \%$ & Shrinkage\% & Value & $\mathrm{CV} \%$ & Value & $\mathrm{CV} \%$ \\
\hline \multicolumn{8}{|l|}{ Fixed effects } \\
\hline $\mathrm{CL}(\mathrm{mL} / \mathrm{min})$ & 32.1 & 5.1 & & 32.3 & 5.2 & 29.9 & 4.8 \\
\hline$V_{1}(\mathrm{~mL})$ & 146 & 42 & & 145 & 32 & 188 & 27 \\
\hline$Q_{2}(\mathrm{~mL} / \mathrm{min})$ & 66.6 & 11 & & 66.8 & 11 & 73.3 & 10 \\
\hline$V_{2}(\mathrm{~mL})$ & 1840 & 6.5 & & 1851 & 6.1 & 1630 & 4.4 \\
\hline$Q_{\text {out }}(\mathrm{mL} / \mathrm{min})$ & 10.8 & N.A. & & 10.8 & N.A. & & \\
\hline$f_{1}$ & 0.0619 & 8.2 & & 0.0621 & 8.3 & & \\
\hline $\mathrm{V}_{\mathrm{Br}}(\mathrm{mL})$ & 269 & 14 & & 281 & 15 & & \\
\hline$f_{2}$ & 8.67 & 2.7 & & 9.51 & 30 & & \\
\hline \multicolumn{8}{|l|}{ Covariates } \\
\hline Kainate (CL) & 0.537 & 8.5 & & 0.539 & 8.4 & 0.556 & 8.8 \\
\hline Kainate $\left(V_{2}\right)$ & 0.678 & 7.0 & & 0.676 & 5.9 & 0.746 & 6.4 \\
\hline Kainate $\left(V_{\mathrm{Br}}\right)$ & 0.592 & 13 & & 0.591 & 12 & & \\
\hline Tariquidar (CL) & 0.835 & 6.8 & & 0.835 & 6.5 & & \\
\hline Tariquidar $\left(Q_{\text {out }}\right)$ & 0.366 & 14 & & 0.376 & 16 & & \\
\hline Tariquidar $\left(Q_{\text {in }}\right)$ & 2.65 & 17 & & 2.73 & 18 & & \\
\hline Tariquidar $\left(f_{2}\right)$ & 5.66 & 33 & & 5.65 & 29 & & \\
\hline Weight (CL) & N.A & N.A & & N.A & N.A & 0.967 & 46 \\
\hline \multicolumn{8}{|c|}{ Inter-individual variability } \\
\hline$\eta(\mathrm{CL})$ & 0.0682 & 20 & 6 & 0.0612 & 19 & 0.0731 & 18 \\
\hline$\eta\left(\mathrm{V}_{1}\right)$ & 1.92 & 30 & 18 & 2.03 & 34 & 1.61 & 29 \\
\hline$\eta\left(\mathrm{V}_{2}\right)$ & 0.0686 & 34 & 15 & 0.680 & 32 & 0.0231 & 50 \\
\hline$\eta\left(\mathrm{Q}_{\text {in }}\right)$ & 0.101 & 19 & 13 & 0.0965 & 19 & & \\
\hline$\eta\left(\mathrm{V}_{\mathrm{br}}\right)$ & 0.196 & 31 & 16 & 0.183 & 44 & & \\
\hline \multicolumn{8}{|c|}{ Propotional residual error } \\
\hline$\sigma^{2}($ plasma $)$ & 0.270 & 7.0 & & 0.271 & 7.6 & 0.233 & 5.6 \\
\hline$\sigma^{2}$ (brain ECF) & 0.185 & 9.4 & & 0.183 & 9.1 & & \\
\hline$\sigma^{2}$ (total brain) & 0.694 & 21 & & 0.636 & 22 & & \\
\hline
\end{tabular}

\section{TQD Treatment}

TQD pre-administration increased $Q_{\text {in }} 2.65$-fold, decreased $Q_{\text {out }} 2.73$-fold (1/0.366) and increased $f_{2}$, i.e., the correlation between $C_{\mathrm{br} 1}$ and $C_{\mathrm{br} 2}, 5.66$-fold (see Table II). Since it was assumed that there was a correlation between $Q_{\text {in }}$ and $Q_{\text {out }}$, the model was also fitted with only TQD influence on either $Q_{\text {in }}$ or $Q_{\text {out }}$, but in both cases the OFV increased by $>25$ units(see Table III). Taken together this indicates that TQD preadministration can increase the free brain concentrations 7.2fold $(2.65 \times 1 / 0.366=7.2)$ by acting on both $Q_{\text {in }}$ and $Q_{\text {out }}$. The increase in total brain concentrations was much larger, about 40fold $(2.65 \times 1 / 0.366 \times 5.66=41)$ indicating that TQD also increased the distribution from the brain ECF to brain tissue.

\section{Kainate Treatment}

Typically, three injections, i.e., a cumulative dose of $20 \mathrm{mg} / \mathrm{kg}$, were needed to reach stage IV or V seizures according to Racine's scale (14) and SE was usually reached within $20 \mathrm{~min}$ after the last injection. Without exception all rats reached this stage. Rats usually displayed seizures for 6$12 \mathrm{~h}$, with the most intense and frequent seizures occurring during the first $2 \mathrm{~h}$. The seizures were not interrupted by any anti-epileptic drug treatment. The mortality was less than $10 \%$. Due to the design of this study it was not possible to determine whether these animals eventually would have developed spontaneous epilepsy, but the signs and symptoms during and after SE were very similar to those observed in previous studies where animals did develop epilepsy. Further, some of the kainate-treated animals had seizures during the microdialysis experiments. The seizures were usually provoked by stress, i.e., the rats experienced seizures during or just after being connected to the perfusion fluid syringe and sample collector.

Kainate treatment was found to be a significant covariate for the systemic clearance (CL) and volume of distribution in the peripheral plasma ( $V_{2}$; see Fig. 2 and Table II). Kainate treatment also reduced the $V_{\mathrm{br}} 1.69$-fold (1/0.59; Table II).

Further, the total brain-to-plasma concentrations ratios at the end of experiments were lower in kainate-treated than in control animals. This brain-to-plasma ratio was (average \pm standard deviation) $0.9 \pm 0.6$ in control animals and $0.6 \pm 0.4$ in kainate-treated animals pre-administered with only vehicle. In TQD pre-administrated animals the ratios were $114 \pm 80$ in control animals and $45 \pm 29$ in kainate-treated animals. However, in the population model kainate as a covariate for $f_{2}$, i.e., the distribution into the deeper brain compartment, did not result in a significant drop of OFV (Table III).

\section{In Vivo Retro Dialysis}

A typical profile of retrodialysis in one animal is shown in Fig. 4. The probe concentration recovery was not 


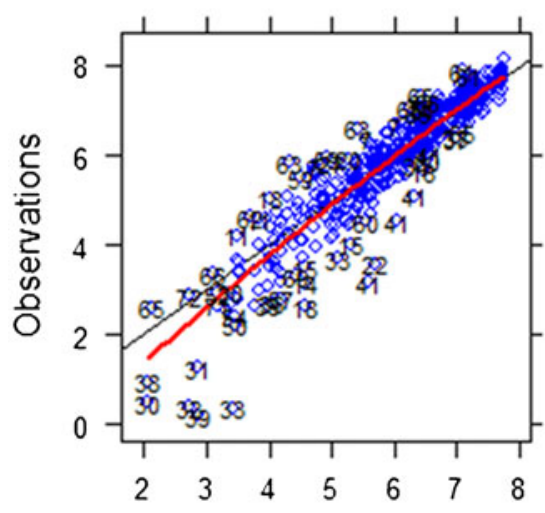

Population predictions

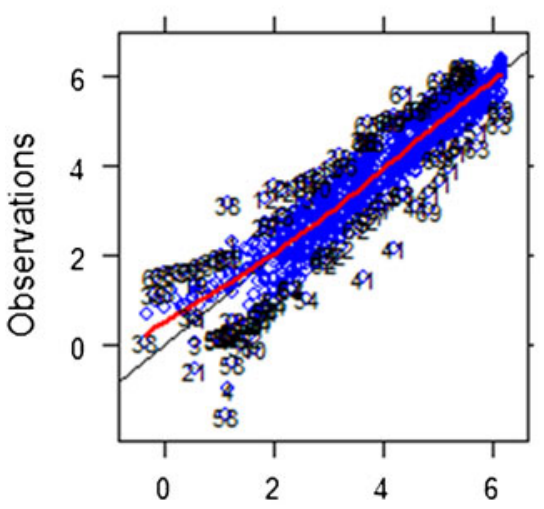

Population predictions

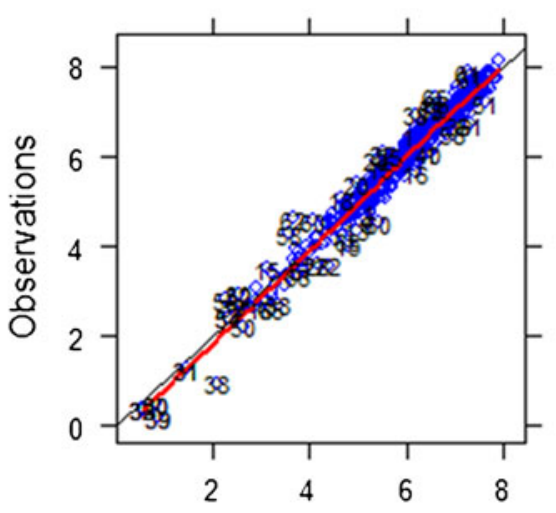

Individual predictions

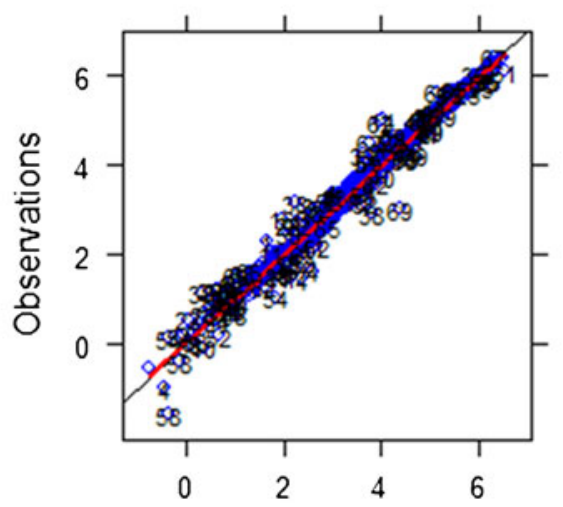

Individual predictions
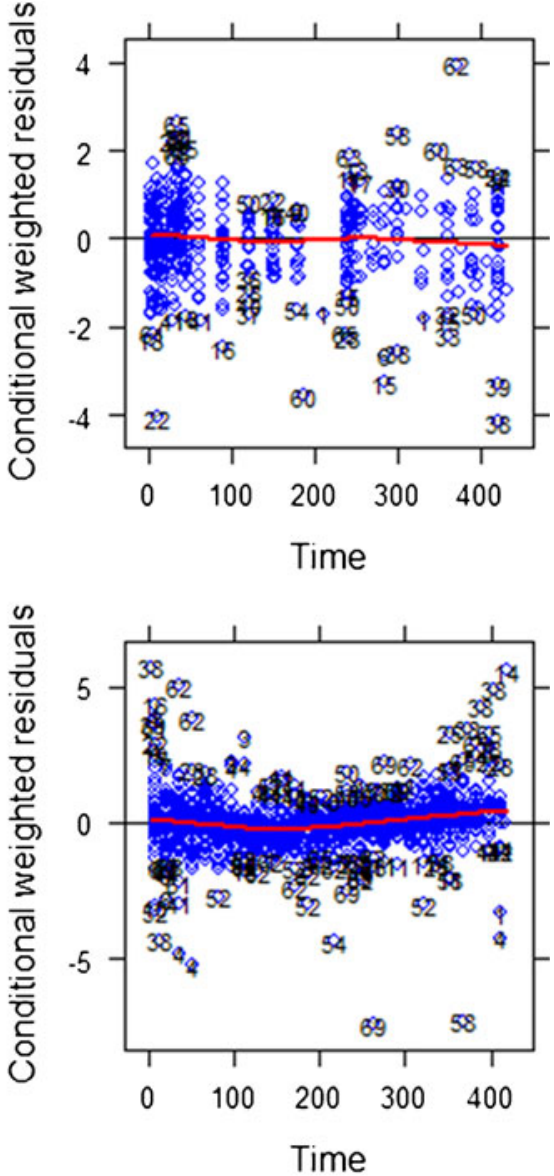

Fig. 3. Model diagnostics for plasma (upper panel) and brain extracellular fluid (lower panel). The model described the data well, as the residuals in the left and middle columns are distributed around the line of identity (thin black line), with dots closer to this line in the middle column as the inter-individual variability is taken into account in these two plots. Plots in the right column show the conditional weighted residuals versus time and these are also randomly scattered around zero, thus showing no trend over time

Table III. Model Development Table

\begin{tabular}{lcl}
\hline \multicolumn{1}{c}{ Model } & $\Delta$ OFV (compared to final model) & Comment \\
\hline 1-comp plasma & 1,133 & Increase $>6.63$ in OFV $\rightarrow$ significant worse fit than final model \\
Without covariates & 40.38 & \\
Kainate $(\mathrm{CL})$ & 14.36 & Increase $>6.63$ in OFV $\rightarrow$ significant worse fit than final model \\
Kainate $\left(V_{2}\right)$ & 9.91 & Increase $>6.63$ in OFV $\rightarrow$ significant worse fit than final model \\
Kainate $\left(V_{\mathrm{Br}}\right)$ & 49.25 & Increase $>6.63$ in OFV $\rightarrow$ significant worse fit than final model \\
Tariquidar $(\mathrm{CL})$ & 28.93 & Increase $>6.63$ in OFV $\rightarrow$ significant worse fit than final model \\
Tariquidar $\left(Q_{\text {out }}\right)$ & 25.41 & Increase $>6.63$ in OFV $\rightarrow$ significant worse fit than final model \\
Tariquidar $\left(Q_{\text {in }}\right)$ & 19.22 & Increase $>6.63$ in OFV $\rightarrow$ significant worse fit than final model \\
Tariquidar $\left(f_{2}\right)$ & & Increase $>6.63$ in OFV $\rightarrow$ significant worse fit than final model \\
With covariates & -0.289 & \\
Weight $(\mathrm{CL})$ & -0.123 & Non-significant change from final model \\
Weight $\left(V_{1}\right)$ & -0.09 & Non-significant change from final model \\
Weight $\left(V_{2}\right)$ & 0.00 & Non-significant change from final model \\
Kainate $(Q)$ & -5.09 & Non-significant change from final model \\
Kainate $\left(V_{1}\right)$ & -5.32 & Non-significant change from final model \\
Kainate $\left(Q_{\text {in }}\right)$ & -6.42 & Non-significant change from final model \\
Kainate $\left(Q_{\text {out }}\right)$ & -0.73 & Non-significant change from final model \\
Kainate $\left(f_{2}\right)$ & -5.19 & Non-significant change from final model \\
Tariquidar $\left(V_{1}\right)$ & -0.02 & Non-significant change from final model \\
Tariquidar $\left(V_{2}\right)$ & -3.11 & Non-significant change from final model \\
Tariquidar $(Q)$ & -2.485 & Non-significant change from final model \\
Tariquidar $\left(V_{\mathrm{Br}}\right)$ & & Non-significant change from final model \\
\hline
\end{tabular}


Table IV. Model Parameter Equations

$$
\begin{aligned}
& \text { Plasma } \\
& \mathrm{CL}=\theta_{1} \cdot \theta_{2}{ }^{\text {KAINATE }} \cdot \theta_{3}{ }^{\text {TARIQUIDAR }} \cdot \exp \left(\eta_{1}\right) \\
& \mathrm{V}_{1}=\theta_{4} \cdot \exp \left(\eta_{2}\right) \\
& \mathrm{Q}=\theta_{5} \\
& \mathrm{~V}_{2}=\theta_{6} \cdot \theta_{7}{ }^{\text {KAINATE }} \cdot \exp \left(\eta_{3}\right) \\
& \text { Brain } \\
& \mathrm{Q}_{\text {out }}=\theta_{8} \cdot \theta_{9} \text { TARIQUIDAR } \\
& \mathrm{V}_{\mathrm{Br}}=\theta_{10} \cdot \theta_{11}{ }^{\text {KAINATE }} \cdot \exp \left(\eta_{4}\right) \\
& \mathrm{f}_{1}=\theta_{12} \\
& \mathrm{Q}_{\text {in }}=\mathrm{f}_{1} \cdot \theta_{8} \cdot \theta_{13}{ }^{\text {TARIQUIDAR }} \cdot \exp \left(\eta_{5}\right) \\
& \mathrm{f}_{2}=\theta_{14} \cdot \theta_{15} \text { TARIQUIDAR }
\end{aligned}
$$

Where covariates KAINATE and TARIQUIDAR are 1 for treated animals and 0 for non-treated animals

dependent on quinidine concentrations (20-500 ng/mL) nor on treatment with TQD. As can be seen in Fig. 4, there was an instantaneous equilibrium (no lag-time) when the concentration in the perfusion fluid was changed. The loss from the perfusion fluid was average $24 \%$ (standard deviation $5.8 \%$ ) and this value was used as the extraction fraction in Eq. 2 to convert quinidine microdialysate concentrations to brain ECF concentrations.

\section{DISCUSSION}

Quinidine concentrations in the brain were very low in both kainate- and saline-treated rats. Remarkably, kainatetreated rats tended to have a lower total brain concentration but a higher brain ECF concentration of quinidine than saline-treated rats. This suggests merely that the P-gp function in kainate-treated rats is altered at the parenchymal level rather than at the BBB. TQD pre-administration increased the brain ECF brain concentration 7.2-fold and the total brain concentration about 40-fold, supporting an

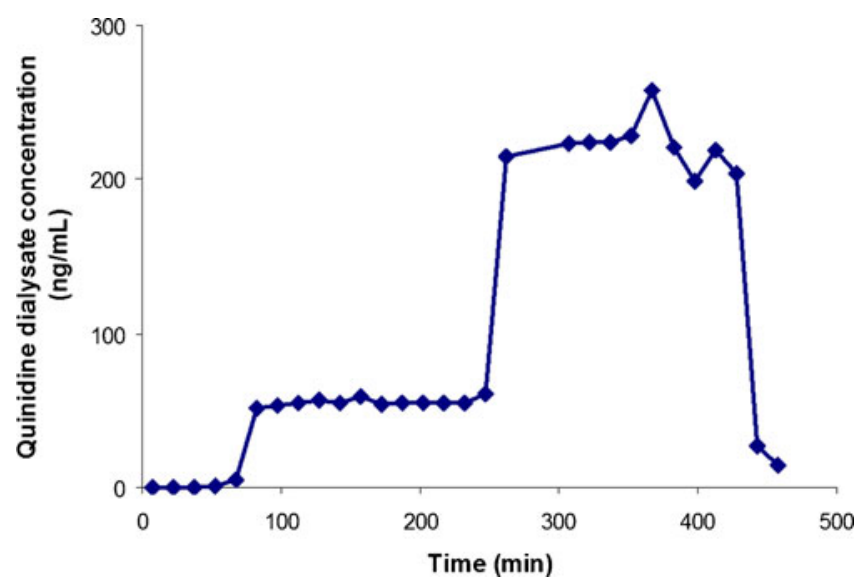

Fig. 4. An in vivo retrodialysis profile for a typical animal. Blank perfusion fluid was perfused during 0-60 min. Then, a perfusion fluid containing $71 \mathrm{ng} / \mathrm{mL}$ quinidine was perfused up to $240 \mathrm{~min}$. From 240 to $420 \mathrm{~min}$, the perfusion solution contained $273 \mathrm{ng} / \mathrm{mL}$ quinidine. After $420 \mathrm{~min}$, the animal was again perfused with blank perfusion fluid. Samples we obtained every $15 \mathrm{~min}$ important role for P-gp in intrabrain distribution. The effect of TQD was similar in control and kainate-treated rats.

Compared to many other methods, microdialysis has the important advantage of measuring the free (ECF) brain concentration $(6,7)$. This concentration is relevant for estimating the transport across the BBB (19). In addition to the free concentrations that were measured with microdialysis over the whole study time, total quinidine concentrations were measured at the end of the experiment. Hence, the combination of microdialysis, plasma and total brain tissue concentrations produced a very rich data set including information on both transport across the $\mathrm{BBB}$ and distribution within the brain.

TQD treatment was used to inhibit the P-gp function. As mentioned, this resulted in a 7.2-fold increase in brain ECFto-plasma concentration ratio and an approximately 40 -fold increase in total brain-to-plasma concentration ratio. PET studies have shown 10-15-fold increase in brain-to-plasma concentrations of the P-gp substrate $(R)-\left[{ }^{11} \mathrm{C}\right]$ verapamil after inhibition with $15 \mathrm{mg} / \mathrm{kg}$ TQD $(11,20)$. PET data can be compared to total concentrations and thus, the present study indicates that quinidine might be a better model drug to study $\mathrm{P}$-gp functionality than verapamil, as a large difference in concentration between baseline and P-gp inhibition states is desirable for a good P-gp function marker, regardless of whether PET or microdialysis is used. Another possible conclusion is that microdialysis as a method is more sensitive than PET to assess changes in P-gp functionality as it measures the free concentration, while the PET signal is not solely reflecting on processes at the BBB but also distribution within the brain. In the future, it would be very interesting to use ${ }^{11} \mathrm{C}$-labeled quinidine as a PET tracer to be able to compare the read-out from microdialysis and PET. Initially, verapamil was also tried in the microdialysis setting, but due to extensive sticking of verapamil to the microdialysis tubing and probes this was not possible. When designing the present study, use of an anti-epileptic drug as a model drug was considered, but since a large difference between normal P-gp function and increased/decreased P-gp function was desired, it was concluded that a strong P-gp substrate, such as quinidine, was the best way to demonstrate proof of concept. In addition, quinidine as opposed to many other Pgp substrates, show no interaction with the other major efflux transporters, e.g., breast cancer resistant protein $(21,22)$.

All brain tissue samples were obtained at approximately the same time point, i.e., at the end of the experiment $7 \mathrm{~h}$ after the start of quinidine infusion, at which equilibrium was assumed to be reached. However, rate constants for distribution to and from the total brain compartment could not be estimated based on the present data set. Therefore, concentration of quinidine in brain tissue was in the final pharmacokinetic model described as a multiple $\left(f_{2}\right)$ of the free brain ECF concentration. Previous studies (unpublished data) in our lab have showed that equilibrium between brain ECF and total brain concentrations is very rapid and hence even if brain samples had been obtained at different time points it would still have been possible to use the present model structure. The result showed that quinidine concentration is 8.7-fold higher in brain tissue than in brain ECF.

In epilepsy, it has been hypothesized that P-gp expression at the $\mathrm{BBB}$ is upregulated and hence contributes to the development of pharmacoresistance (23-28). Therefore, it 
was also of interest to investigate the P-gp function in kainate-treated rats, a frequently used model for temporal lobe epilepsy, and compare quinidine pharmacokinetics in this epilepsy model both at baseline and after P-gp inhibition to naïve control rats. Rats subjected to kainate induced SE become truly epileptic only after 3-4 weeks when spontaneous seizures start to occur (8), but the most prominent increase in P-gp expression has been reported between 1 and 7 days after SE (9,10,29-31). Thus, although seizures were not yet present at 1 week after induction of SE by kainate (apart from a few incidental observations), it can be assumed that at this time point after SE the brain both exhibits increased P-gp expression and is progressing towards the epileptic state. In a previously published study using the same epilepsy model, it was shown that the brain glucose metabolism, measured with ${ }^{18}$ F-fluorodeoxyglucose, was significantly lower in kainate-treated rats 7 days post-SE than in controls (11). It is a common clinical observation that ${ }^{18} \mathrm{~F}$-fluorodeoxyglucose uptake is decreased in epileptic brain tissue (32). This suggests that the kainate model in rats mimics some functional changes that have been observed in the human epileptic brain.

Kainate treatment influenced the plasma pharmacokinetics of quinidine by decreasing the clearance (CL) and the peripheral volume of distribution $\left(V_{2}\right)$. There was no effect on the $Q_{\text {in }}$ and $Q_{\text {out }}$, but kainate treatment decreased the volume of distribution in the brain, $V_{\mathrm{br}}$. A decrease in $V_{\mathrm{br}}$ suggests higher concentrations of quinidine in the brain, which may appear to be a contradiction to the notion that kainate treatment should result in upregulation of P-gp and thus rather a decrease in brain concentrations. However, P-gp is not only expressed at the endothelial cells of the BBB but also at other cell types such as astrocytes and pericytes (33-35). There are studies showing that breakdown of $\mathrm{BBB}$ function may be a consequence of an inflammatory response and that inflammation is a cause for P-gp upregulation (33). In the normal healthy brain, P-gp is probably not an important player in the brain beyond the endothelial cells, but after SE, an inflammatory reaction could lead to upregulation of P-gp not only in endothelial cells, but also in glial cells and neurons and these may thus act as a "second line of defense in addition to the BBB" (34-36). Total brain concentrations appeared to be lower in kainate-treated although this effect did not reach significance in this study, probably due to a large variability in the different rat groups. In this context, the findings of Bankstahl et al. are of interest, as they reported that, after SE, total brain-to-plasma ratio of phenytoin in rats was lower, while the opposite was found in the brain dialysate to plasma ratio (30). Phenytoin is considered to be a (weak) P-gp substrate (37) and hence the results by Bankstahl et al. are in line with the results from the present study, i.e., that SE decreases the total brain concentrations and increases the free brain concentrations.

\section{CONCLUSION}

Quinidine uptake in the brain was very low in both kainateand saline-treated rats. The effect of TQD was similar in control and kainate-treated rats and TQD treatment increased total quinidine brain concentrations more than ECF concentrations. Kainate-treated rats tended to have a lower total brain concentration and a higher brain ECF concentration of quinidine than saline-treated rats. Thus, this study did not provide evidence for the hypothesis that $\mathrm{P}$-gp function is altered at the endothelial capillaries constituting the BBB at 1 week after SE induction. However, the study indicated that P-gp functionality might be altered at the brain parenchyma.

\section{ACKNOWLEDGMENTS}

We thank Robin Hartman, Maaike Labots, and Nathalie Doorenweerd for help with the animal surgery and microdialysis experiments. This work was supported by the EU 7th framework program EURIPIDES (FP7/2007-2013 under grant agreement no. 201380).

Open Access This article is distributed under the terms of the Creative Commons Attribution Noncommercial License which permits any noncommercial use, distribution, and reproduction in any medium, provided the original author(s) and source are credited.

\section{REFERENCES}

1. Schinkel AH, Jonker JW. Mammalian drug efflux transporters of the ATP binding cassette (ABC) family: an overview. Adv Drug Deliv Rev. 2003;55:3-29.

2. Cordon-Cardo C, O'Brien JP, Casals D, Rittman-Grauer L, Biedler JL, Melamed MR, Bertino JR. Multidrug-resistance gene (P-glycoprotein) is expressed by endothelial cells at blood-brain barrier sites. Proc Natl Acad Sci U S A. 1989;86:695-8.

3. Schmidt D, Löscher W. Drug resistance in epilepsy: putative neurobiologic and clinical mechanisms. Epilepsia. 2005;46:85877.

4. Regesta G, Tanganelli P. Clinical aspects and biological bases of drug-resistant epilepsies. Epilepsy Res. 1999;34:109-22.

5. Hammarlund-Udenaes M, Bredberg U, Friden M. Methodologies to assess brain drug delivery in lead optimization. Curr Top Med Chem. 2009;9:148-62.

6. de Lange EC, Danhof M, de Boer AG, Breimer DD. Methodological considerations of intracerebral microdialysis in pharmacokinetic studies on drug transport across the blood-brain barrier. Brain Res Rev. 1997;25:27-49.

7. de Lange EC, de Boer AG, Breimer DD. Methodological issues in microdialysis sampling for pharmacokinetic studies. Adv Drug Deliv Rev. 2000;45:125-48.

8. Hellier JL, Patrylo PR, Buckmaster PS, Dudek FE. Recurrent spontaneous motor seizures after repeated low-dose systemic treatment with kainate: assessment of a rat model of temporal lobe epilepsy. Epilepsy Res. 1998;31:73-84.

9. Zhang L, Ong WY, Lee T. Induction of P-glycoprotein expression in astrocytes following intracerebroventricular kainate injections. Exp Brain Res. 1999;126:509-16.

10. Seegers U, Potschka H, Loscher W. Transient increase of Pglycoprotein expression in endothelium and parenchyma of limbic brain regions in the kainate model of temporal lobe epilepsy. Epilepsy Res. 2002;51:257-68.

11. Syvänen S, Luurtsema G, Molthoff CF, Windhorst AD, Huisman MC, Lammertsma AA, Voskuyl RA, de Lange EC. $(R)-\left[{ }^{11} \mathrm{C}\right]$ Verapamil PET studies to assess changes in P-glycoprotein expression and functionality in rat blood-brain barrier after exposure to kainate-induced status epilepticus. BMC. 2011;11:1.

12. Moghaddam B, Bunney BS. Ionic composition of microdialysis perfusing solution alters the pharmacological responsiveness and basal outflow of striatal dopamine. J Neurochem. 1989;53:652-4.

13. Chaurasia CS, Muller M, Bashaw ED, Benfeldt E, Bolinder J, Bullock R, Bungay PM, DeLange EC, Derendorf $\mathrm{H}$, Elmquist WF, Hammarlund-Udenaes M, Joukhadar C, Kellogg Jr DL, Lunte CE, Nordstrom CH, Rollema $\mathrm{H}$, Sawchuk RJ, Cheung BW, Shah VP, Stahle L, Ungerstedt U, Welty DF, Yeo H. AAPS-FDA workshop white paper: 
microdialysis principles, application and regulatory perspectives. Pharm Res. 2007;24:1014-25.

14. Racine R, Okujava V, Chipashvili S. Modification of seizure activity by electrical stimulation. 3. Mechanisms. Electroencephalogr Clin Neurophysiol. 1972;32:295-9.

15. Le Quellec A, Dupin S, Genissel P, Saivin S, Marchand B, Houin G. Microdialysis probes calibration: gradient and tissue dependent changes in no net flux and reverse dialysis methods. J Pharmacol Toxicol Methods. 1995;33:11-6.

16. Jonsson EN, Karlsson MO. Xpose-an S-PLUS based population pharmacokinetic/pharmacodynamic model building aid for NONMEM. Comput Methods Prog Biomed. 1999;58:51-64.

17. Wilkins JJ. NONMEMory: a run management tool for NONMEM. Comput Methods Prog Biomed. 2005;78:259-67.

18. Hooker AC, Staatz CE, Karlsson MO. Conditional weighted residuals (CWRES): a model diagnostic for the FOCE method. Pharm Res. 2007;24:2187-97.

19. Hammarlund-Udenaes M, Friden M, Syvanen S, Gupta A. On the rate and extent of drug delivery to the brain. Pharm Res. 2008;25:1737-50.

20. Bankstahl JP, Kuntner C, Abrahim A, Karch R, Stanek J, Wanek T, Wadsak W, Kletter K, Muller M, Löscher W, Langer O. Tariquidar-induced P-glycoprotein inhibition at the rat bloodbrain barrier studied with $(R)-{ }^{11} \mathrm{C}$-verapamil and PET. J Nucl Med. 2008;49:1328-35.

21. Kodaira H, Kusuhara H, Fujita T, Ushiki J, Fuse E, Sugiyama Y. Quantitative evaluation of the impact of active efflux by $p$ glycoprotein and breast cancer resistance protein at the bloodbrain barrier on the predictability of the unbound concentrations of drugs in the brain using cerebrospinal fluid concentration as a surrogate. J Pharmacol Exp Ther. 2011;339:935-44.

22. Matsson P, Pedersen JM, Norinder U, Bergstrom CA, Artursson P. Identification of novel specific and general inhibitors of the three major human ATP-binding cassette transporters Pgp, BCRP and MRP2 among registered drugs. Pharm Res. 2009;26:1816-31.

23. Potschka H. Targeting regulation of ABC efflux transporters in brain diseases: a novel therapeutic approach. Pharmacol Ther. 2009;125:118-27.

24. Zibell G, Unkruer B, Pekcec A, Hartz AM, Bauer B, Miller DS, Potschka H. Prevention of seizure-induced up-regulation of endothelial P-glycoprotein by COX-2 inhibition. Neuropharmacology. 2009;56:849-55.

25. Sisodiya SM, Lin WR, Harding BN, Squier MV, Thom M. Drug resistance in epilepsy: expression of drug resistance proteins in common causes of refractory epilepsy. Brain. 2002;125:22-31.

26. Tishler DM, Weinberg KI, Hinton DR, Barbaro N, Annett GM, Raffel C. MDR1 gene expression in brain of patients with medically intractable epilepsy. Epilepsia. 1995;36:1-6.

27. Dombrowski SM, Desai SY, Marroni M, Cucullo L, Goodrich $\mathrm{K}$, Bingaman W, Mayberg MR, Bengez L, Janigro D. Overexpression of multiple drug resistance genes in endothelial cells from patients with refractory epilepsy. Epilepsia. 2001;42:1501-6.

28. Löscher W, Potschka H. Role of drug efflux transporters in the brain for drug disposition and treatment of brain diseases. Prog Neurobiol. 2005;76:22-76.

29. van Vliet E, Aronica E, Redeker S, Marchi N, Rizzi M, Vezzani A, Gorter J. Selective and persistent upregulation of mdr1b mRNA and P-glycoprotein in the parahippocampal cortex of chronic epileptic rats. Epilepsy Res. 2004;60:203-13.

30. Bankstahl JP, Löscher W. Resistance to antiepileptic drugs and expression of P-glycoprotein in two rat models of status epilepticus. Epilepsy Res. 2008;82:70-85.

31. Kuteykin-Teplyakov K, Brandt C, Hoffmann K, Loscher W. Complex time-dependent alterations in the brain expression of different drug efflux transporter genes after status epilepticus. Epilepsia. 2009;50:887-97.

32. Henry TR, Votaw JR. The role of positron emission tomography with $\left[{ }^{18} \mathrm{~F}\right]$ fluorodeoxyglucose in the evaluation of the epilepsies. Neuroimaging Clin N Am. 2004;14:517-35.

33. Ronaldson PT, Persidsky Y, Bendayan R. Regulation of ABC membrane transporters in glial cells: relevance to the pharmacotherapy of brain HIV-1 infection. Glia. 2008;56:1711-35.

34. Wolburg H, Noell S, Mack A, Wolburg-Buchholz K, FallierBecker P. Brain endothelial cells and the glio-vascular complex. Cell Tissue Res. 2009;335:75-96.

35. Shimizu F, Sano Y, Maeda T, Abe MA, Nakayama H, Takahashi R, Ueda M, Ohtsuki S, Terasaki T, Obinata M, Kanda T. Peripheral nerve pericytes originating from the blood-nerve barrier expresses tight junctional molecules and transporters as barrier-forming cells. J Cell Physiol. 2008;217:388-99.

36. Roberts DJ, Goralski KB. A critical overview of the influence of inflammation and infection on P-glycoprotein expression and activity in the brain. Expert Opin Drug Metab Toxicol. 2008:4:1245-64.

37. Potschka H, Löscher W. In vivo evidence for P-glycoproteinmediated transport of phenytoin at the blood-brain barrier of rats. Epilepsia. 2001;42:1231-40. 